\title{
An intriguing solar microflare observed with RHESSI, Hinode, and TRACE
}

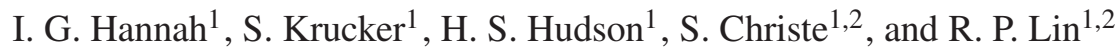 \\ 1 Space Sciences Laboratory, University of California at Berkeley, Berkeley, CA, 94720-7450, USA \\ e-mail: hannah@ssl. berkeley.edu \\ 2 Physics Department, University of California at Berkeley, Berkeley, CA, 94720-7450, USA
}

Received 8 November 2007 / Accepted 3 December 2007

\section{ABSTRACT}

\begin{abstract}
Aims. We investigate particle acceleration and heating in a solar microflare.
Methods. In a microflare with non-thermal emission to remarkably high energies ( $>50 \mathrm{keV})$, we investigate the hard X-rays with RHESSI imaging and spectroscopy and the resulting thermal emission seen in soft X-rays with Hinode/XRT and in EUV with TRACE.

Results. The non-thermal footpoints observed with RHESSI spatially and temporally match bright footpoint emission in soft X-rays and EUV. There is the possibility that the non-thermal spectrum extends down to $4 \mathrm{keV}$. The hard X-ray burst clearly does not follow the expected Neupert effect, with the time integrated hard X-rays not matching the soft X-ray time profile. So, although this is a simple microflare with good X-ray observation coverage it does not fit the standard flare model.
\end{abstract}

Key words. Sun: corona - Sun: flares - Sun: X-rays, gamma rays

\section{Introduction}

A Solar flare is the observed multi-wavelength atmospheric response to a rapid transient release of magnetic energy from coronal fields. This process is observed over many scales with small flares, namely microflares, exhibiting energies expressed as millionths of that in the largest flares. The general scenario of a flare is that accelerated particles leave a coronal region where they have gained energy liberated from the coronal magnetic fields, facilitated by magnetic reconnection. These particles stream along the magnetic fields and are seen in hard X-rays via thick target collisional bremsstrahlung at the loop footpoints where the local density is large enough to stop the particles. As well as creating hard X-rays, these accelerated electrons heat the local plasma which evaporates into coronal loops, seen initially in soft X-rays and eventually in EUV as the material cools. The Neupert effect, the time integrated hard (non-thermal) X-ray time profile matching the soft (thermal) X-ray time profile, is often taken as a signature of this behavior (Neupert 1968; Hudson \& Ohki 1972; Dennis \& Zarro 1993; Veronig et al. 2005).

In this paper we present an analysis into this scenario using a GOES A-Class microflare where the Neupert effect is not observed, despite good hard and soft X-ray coverage: the event was observed in soft and hard X-rays with RHESSI (Lin et al. 2002), the X-ray telescope XRT (Golub et al. 2007) on Hinode (Kosugi et al. 2007) and in EUV with TRACE (Handy et al. 1999). The microflare analyzed in this paper starts at 05:13:28 November 17,2006 and shows a remarkably hard/flat spectrum to high energies $(>50 \mathrm{keV})$. This is unusual as microflares observed with RHESSI typically have soft/steep spectra observable only up to about $25 \mathrm{keV}$ (Hannah et al. 2008). This microflare is from active region AR10923 which produced several hard microflares, with a partially occulted one suggestive of thin target emission (Krucker et al. 2007). This active region also produced many type III radio bursts, a further signature of accelerated electrons (Bastian et al. 1998).

\section{Observation and data analysis}

There is uninterrupted RHESSI coverage over the whole of this microflare. However by November 2006, radiation damage to RHESSI's detectors had resulted in an increased background and degraded spectral resolution. This damage affects each of the 9 detectors differently and so we have tried to use those least affected in the analysis: detectors 1, 4, 6 for spectral analysis and detectors 3, 4, 6, 8, 9 for imaging. The damage still influences the results, particularly the spectral analysis below $10 \mathrm{keV}$, discussed further in Sect. 2.1. During the microflare there are XRT images every $20 \mathrm{~s}$, alternating between the C-Poly and Ti-Poly filters, with exposure times between 0.1 and $0.5 \mathrm{~s}$. The Ti-Poly were taken using a lower resolution $\left(2^{\prime \prime}\right)$ compared to the C-Poly images $\left(1^{\prime \prime}\right)$. These filters have similar temperature responses, mostly sensitive to $<10 \mathrm{MK}$ plasma. TRACE images are also available for this event, with the $195 \AA$ and $284 \AA$ filters. The $195 \AA$ images are every $\approx 45 \mathrm{~s}$ over this event, whereas only two $284 \AA$ images are available, one pre-flare, the other during the flare. In addition there was also a bright type III radio burst associated with the flare impulsive peak observed by various radio instruments, such as WIND/WAVES (Bougeret et al. 1995), STEREO/WAVES (Bougeret et al. 2007) and Culgoora. A subsequent increase in interplanetary energetic particles was also observed about $30 \mathrm{~min}$ after the microflare with WIND/3DP (Lin et al. 1995). 


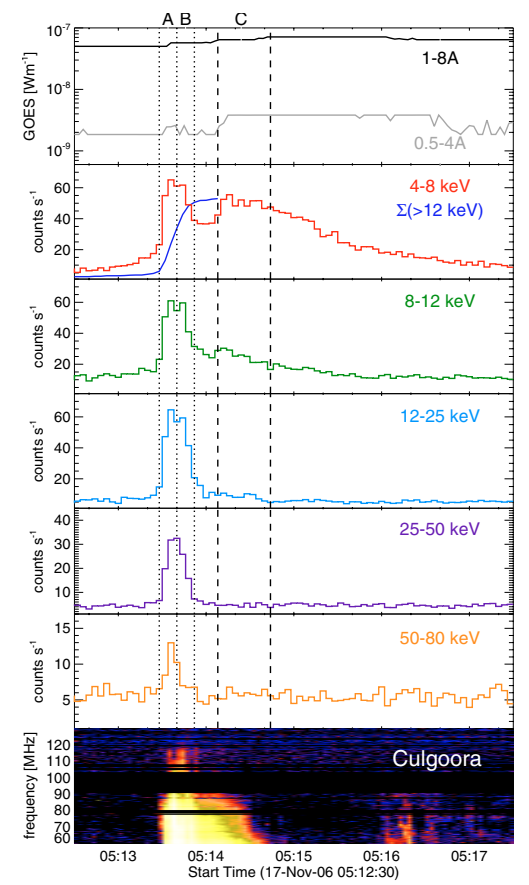

Fig. 1. X-ray and radio time profiles for the 05:13 November 17, 2006 microflare, showing the GOES soft X-ray emission and RHESSI observations in five energy ranges. The $4-8 \mathrm{keV}$ lightcurve is overplotted with the scaled integrated non-thermal emission $>12 \mathrm{keV}$. The vertical dotted and dashed lines indicate three time periods for which the RHESSI images and spectra have been analyzed: two $12 \mathrm{~s}$ impulsive intervals (A and B) and a $36 \mathrm{~s}$ thermal period $(\mathrm{C})$. The spectrogram in the bottom panel is Culgoora radio data, showing a strong type III burst.

\subsection{Time profiles and spectra}

The X-ray and radio time profiles for the microflare are shown in Fig. 1. The GOES soft X-ray lightcurve peaks at GOES A7 level in the 1-8 $\AA$ channel, or A2 level taking the pre-flare background into account. At the lowest RHESSI energies (4-8 keV), there are 2 distinctive peaks of emission: a short impulsive one from $05: 13: 28$ to $05: 13: 52$ and a second from 05:14:08 until nearly $05: 17: 00$. The higher energy ranges $(>12 \mathrm{keV})$ only show emission during the initial impulsive phase. This impulsive initial emission extends to remarkably high energies $(>50 \mathrm{keV})$ and is closely associated with a type III radio burst, shown in the bottom panel of Fig. 1. These factors clearly indicate that above $12 \mathrm{keV}$ the emission is mostly non-thermal. Normally the lowest energies RHESSI observes are predominantly thermal, but the time profile of the $4-8 \mathrm{keV}$ emission does not demonstrate the Neupert effect: the time integrated non-thermal emission $>12 \mathrm{keV}$, shown in Fig. 1, does not match the observed peak in 4-8 keV. This implies that either the $4-8 \mathrm{keV}$ emission during the impulsive peak is non-thermal or some other process is occurring other than simple evaporation and heating. Although in the vast majority of flares the low energy RHESSI observes is thermal in origin, non-thermal emission dominating at these energies has been suggested in a previous event (Sui et al. 2006).

To investigate this in detail, the RHESSI spectra during three time intervals have been analyzed: A $05: 13: 28$ to $05: 13: 40$, the rise phase of the impulsive period, B $05: 13: 40$ to $05: 13: 52$ the decay phase of the impulsive period, and C $05: 14: 08$ to $05: 14: 44$ the second peak. All the spectra during these time intervals have been fitted with a thermal plus broken power-law (to represent the non-thermal emission) model, shown in Fig. 2. Additionally, the first two spectra, A, B during the impulsive peak, have also been fitted by just a broken power-law spectrum, indicated by the upper green line fit and multiplied by a factor of 10 to separate it from the other model fit shown. The resulting photon spectra appear different for the two model fits as the conversion from observed counts in the detectors to photons depends on the fitted model convolved with RHESSI's non-diagonal detector response matrix. This means that in the photon spectrum fitted with a thermal component the thermal line features are overemphasized. Normally the existence of a thermal component is trivial to determine as the thermal line features are directly detectable in the counts spectrum, indicating plasma $\geq 8 \mathrm{MK}$ (Phillips 2004), but due to the RHESSI's radiation-damaged detectors these are not apparent. This damage also skews the resulting thermal fit, producing slightly higher temperatures and lower emission measures than expected (Hannah et al. 2008). This resulting uncertainty causes an ambiguity in the transition from where the the thermal dominates over the non-thermal component and so the broken power-law is fitted using a fixed break energy of $12 \mathrm{keV}$. The spectra are however, definitely very flat/hard above this break, with $\gamma \approx 2.3-2.4$.

Assuming the power-law emission is thick-target the power in the electrons can be estimated via Brown (1971): the power in electrons above $12 \mathrm{keV}$ is $4 \times 10^{26} \mathrm{erg} \mathrm{s}^{-1}$ and $3 \times 10^{26} \mathrm{erg} \mathrm{s}^{-1}$ over time intervals A and B. Over each of these $12 \mathrm{~s}$ time periods the total non-thermal energy is $8 \times 10^{27} \mathrm{erg}$. The purely non-thermal broken power-law model produces slightly flatter spectrum, though still very steep with $\gamma \approx 2.6-2.5$, with breaks down to around $4 \mathrm{keV}$. The power in electrons above $4 \mathrm{keV}$ in these spectra is $2 \times 10^{27} \mathrm{erg} \mathrm{s}^{-1}$ and $3 \times 10^{27} \mathrm{erg} \mathrm{s}^{-1}$ over intervals $\mathrm{A}$ and $\mathrm{B}$, estimating the total non-thermal energy as $5 \times 10^{28} \mathrm{erg}$. The difference of a factor $\approx 6$ in the total non-thermal energy is a guide to the systematic errors in the estimate.

\subsection{X-ray and EUV imaging}

The time progressions of the RHESSI and XRT X-ray images for the microflare are shown in Fig. 3. Pre-flare (05:13:14), XRT observes 2 curved loops with their left footpoints a few arcseconds apart and the right footpoints brighter and unresolvably close together. When the flare begins the left footpoints in XRT brighten and RHESSI observes, over time interval A, a single non-thermal $12-60 \mathrm{keV}$ footpoint at this location, as well as 4-8 keV emission across the XRT loop. As the impulsive phase of the flare declines, time interval $\mathrm{B}$, both left and right footpoints are bright in XRT, with the emission saturating at the right footpoints. The RHESSI $4-8 \mathrm{keV}$ emission again covers the XRT loop and now two $12-60 \mathrm{keV}$ footpoints match the two bright XRT sources. During time interval C only the $4-8 \mathrm{keV}$ RHESSI emission is imageable, producing a single source that matches the brightening righthand side of the loops seen in XRT. After the flare emission ends in RHESSI, the two hot bright loops are still visible in XRT, slightly further apart than pre-flare.

The $195 \AA$ TRACE images of this microflare are shown in Fig. 4. Pre-flare no noticeable counterpart structures to those seen in XRT are observed. At later stages of the flare and postflare similar behavior is observed to that in XRT but delayed. Namely the left footpoints initially brighten with the later emission occurring from the righthand side of the loops. This is consistent with the hot material initially seen by RHESSI and XRT, eventually cooling below $2 \mathrm{MK}$ and being seen in EUV. There is also a $284 \AA$ TRACE image during the decline phase of the RHESSI impulsive peak, time interval B. This image shows two 

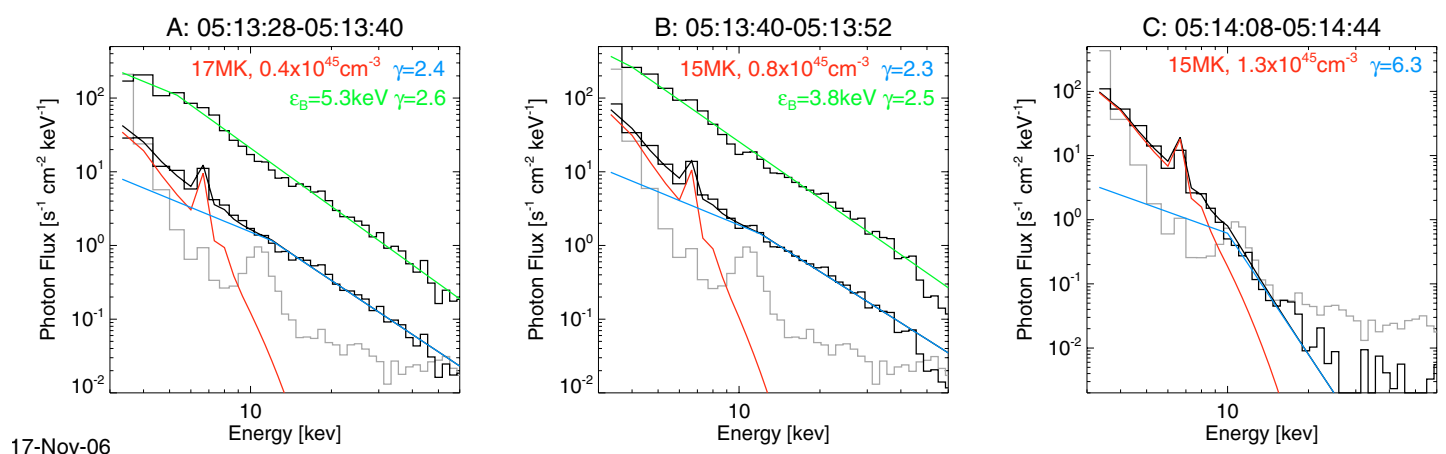

Fig. 2. RHESSI hard X-ray spectra for the three time intervals shown in Fig. 1. The black and grey binned lines indicate the background subtracted data and the background emission. The red, blue and black lines show the thermal, broken power-law and total (thermal plus broken power-law) model. The top data line fitted with a green line in the first two spectra is the data fitted with just a broken power-law model, multiplied by 10 to make it distinguishable from the other spectrum. The photon spectra are different for the two fits in the same time interval because they are found from the same count spectra convolved with RHESSI's detector response and the different model fits.
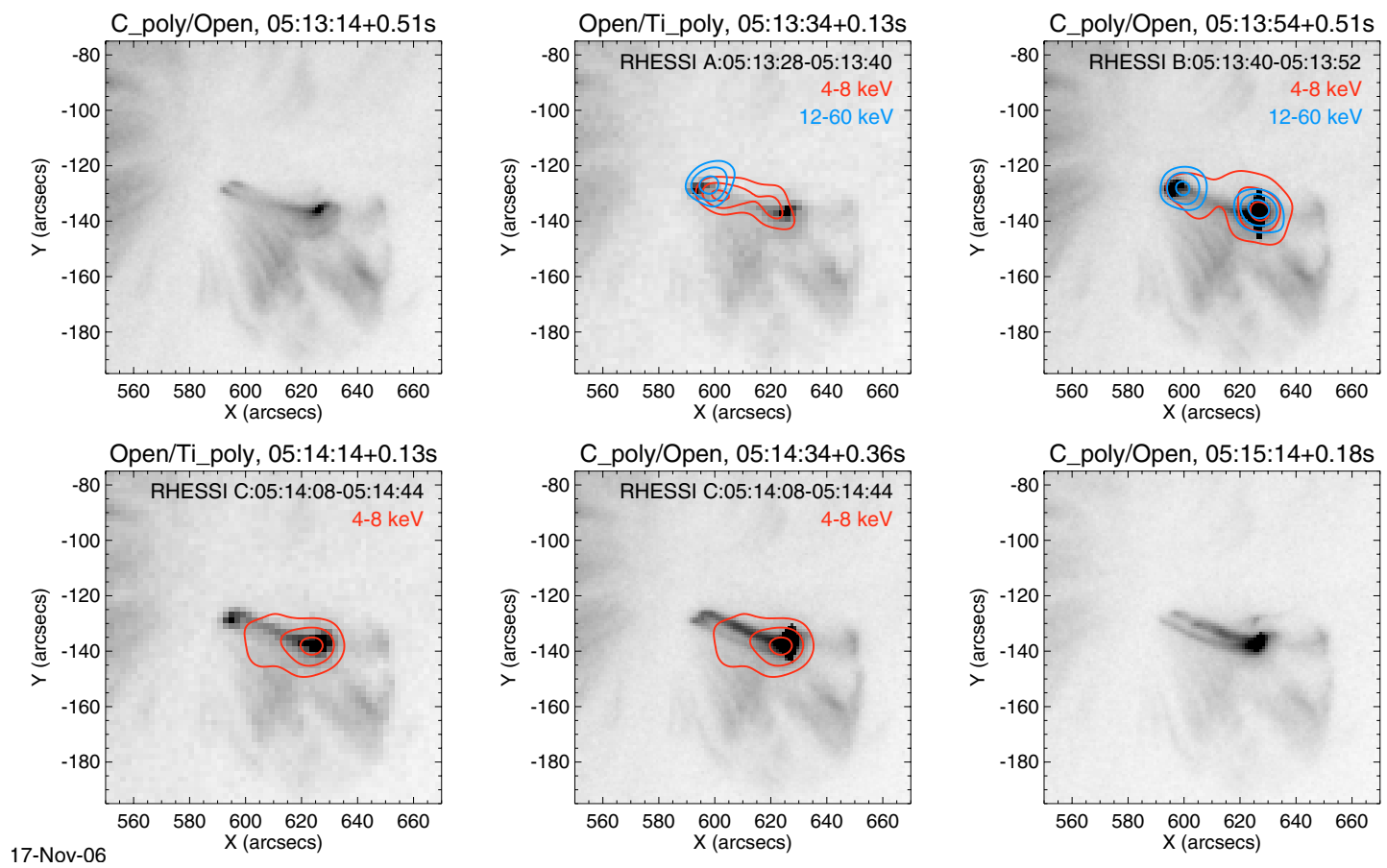

Fig. 3. X-ray imaging at six different times during the 05:13 November 17, 2006 microflare: (top left) pre-flare (top middle) first 12 second impulsive period (top right) second $12 \mathrm{~s}$ impulsive period (bottom left and middle) 36 second of thermal period and (bottom right) post-flare. The background images are XRT with either the C-Poly or Ti-Poly filters, the latter using a lower resolution. The number after the time in the title of each image is the exposure time of each XRT image. The overplotted contours $(50,70,90 \%)$ are the RHESSI images reconstructed using the CLEAN algorithm with detectors 3, 4, 6, 8 and 9 . The red contours represent the 4-8 keV emission (predominantly thermal) and the blue contours are the 12-60 keV emission (mostly non-thermal). The pointing of all the XRT images have been correct by $x+5^{\prime \prime}$ and $y+8^{\prime \prime}$. This was found by matching the RHESSI hard X-ray footpoints with XRT bright emission in the trop right image.

bright footpoints that closely match the bright $12-60 \mathrm{keV}$ nonthermal footpoints seen in RHESSI. This is indicative of the accelerated electrons, inferred by the RHESSI observations, producing continuum observable in TRACE with the $284 \AA$ filter.

\section{Discussion and conclusions}

The microflare presented here clearly shows electrons accelerated to relatively high energies, resulting in heating that is observed in X-rays and EUV. The lower energy RHESSI observations however do not demonstrate the expected Neupert effect in their time profile, suggesting that the initial 4-8 keV emission may not be thermal. Such hard flat spectra with unexpectedly delayed thermal emission have been observed previously in early impulsive flares (Farnik et al. 1997; Sui et al. 2007) but never in such a small flare or with possible non-thermal emission to low energies $(<8 \mathrm{keV})$. An alternative to the standard flare scenario for this microflare is that the non-thermal emission observed in the initial impulsive peak deposits its energy, resulting in chromospheric evaporation, but these higher energy electrons penetrate deeper into the chromosphere, cooling quickly in the dense lower chromosphere, resulting in a diminished upward flow (McDonald et al. 1999). This still leaves the low energy $(<8 \mathrm{keV})$ accelerated electron population, where the bulk of the non-thermal energy input lies, which should produce 

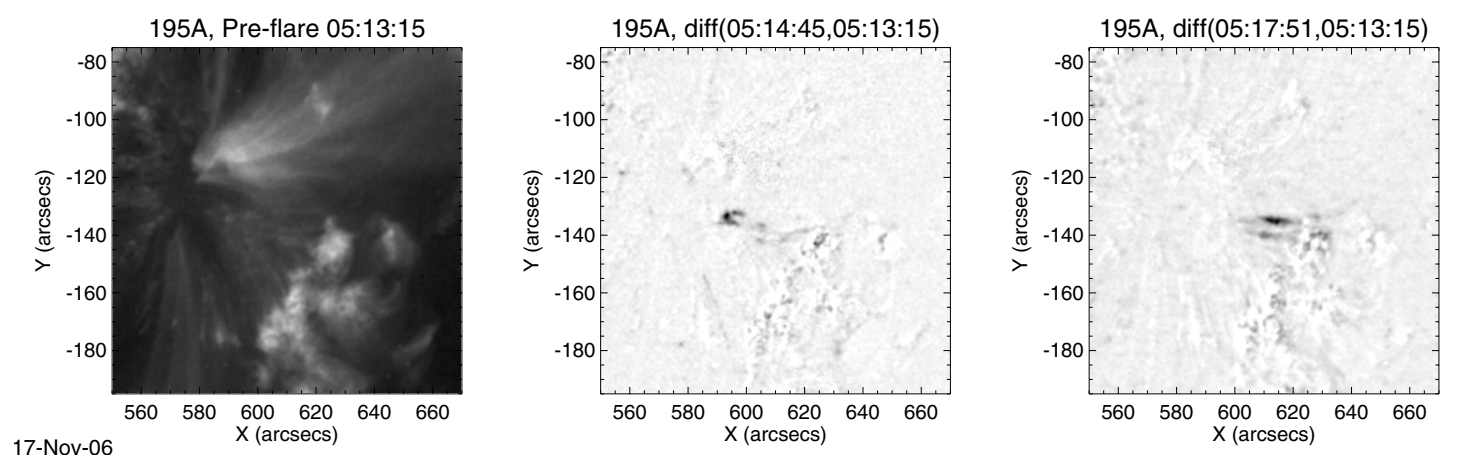

Fig. 4. TRACE $195 \AA$ A EUV images of the 05:13 November 17, 2006 microflare: (left) pre-flare image (middle) difference image between an image during the RHESSI thermal period and the pre-flare image (right) difference image between post- and the pre-flare images. The normalization and order of the color scales varies between the pre-flare image and the difference images.

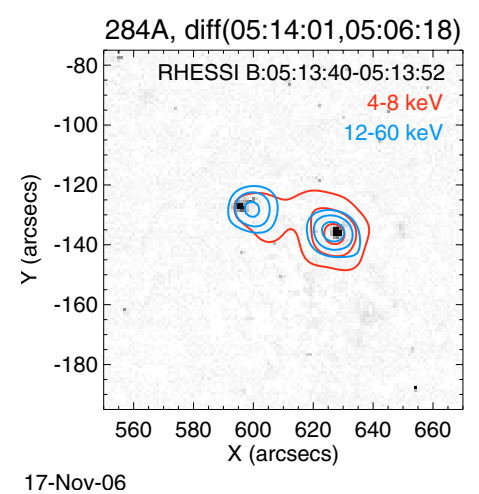

Fig. 5. TRACE $284 \AA$ EUV difference image during the 05:13 November 17, 2006 microflare. The difference image is between an image just after the impulsive period and the pre-flare. Overplotted are the RHESSI thermal (4-8 keV) and non-thermal (12-60 keV) contours from impulsive interval $\mathrm{B}$. The pointing of the TRACE image has been correct by $x+3^{\prime \prime}$ and $y+7^{\prime \prime}$. This was found by matching the RHESSI hard X-ray footpoints with bright EUV footpoints.

detectable evaporation. This could be responsible for the subsequent thermal emission, but the delay is still intriguing and requires further study and modeling.

One major consequence of the non-thermal emission extending down to lower energies is an increased non-thermal energy input into the lower atmosphere. If we assume that this non-thermal energy is deposited into approximately the footpoint area in the XRT images, then the electron fluxes can be estimated. During both time interval A and B, the individual XRT footpoint area is about $4^{\prime \prime} \times 6^{\prime \prime}$ or $\lesssim 10^{17} \mathrm{~cm}^{2}$. With a thermal component at the lowest RHESSI energies then the fluxes of electrons with $>12 \mathrm{keV}$ deposited is $4 \times 10^{9} \mathrm{erg} \mathrm{cm}^{-2} \mathrm{~s}^{-1}$, into a single footpoint during interval $\mathrm{A}$, and $2 \times 10^{9} \mathrm{erg} \mathrm{cm}^{-2} \mathrm{~s}^{-1}$, in total into the two footpoints during interval B. Both of these values are indicative of gentle chromospheric evaporation (Fisher et al. 1985; Abbett \& Hawley 1999; Brosius \& Phillips 2004; Milligan et al. 2006) since the fluxes of flare-accelerated electrons are $\leq 10^{10} \mathrm{erg} \mathrm{cm}^{-2} \mathrm{~s}^{-1}$. If instead the RHESSI emission is purely non-thermal down to low energies then the fluxes of electrons with $>4 \mathrm{keV}$ deposited is $2 \times 10^{10} \mathrm{erg} \mathrm{cm}^{-2} \mathrm{~s}^{-1}$ and $1 \times 10^{10} \mathrm{erg} \mathrm{cm}^{-2} \mathrm{~s}^{-1}$. These values are larger and could lie in the regime of explosive evaporation $\geq 3 \times 10^{10} \mathrm{erg} \mathrm{cm}^{-2} \mathrm{~s}^{-1}$. During the second peak (interval $C$ ) the RHESSI time profile and spectrum are more consistent with thermal emission. Using the RHESSI emission measure from this time $\left(1.3 \times 10^{45} \mathrm{~cm}^{-3}\right)$ and estimating the loop volume from the XRT image, $\left(32^{\prime \prime} \times 3^{\prime \prime}\right)$ giving $10^{26} \mathrm{~cm}^{-3}$, the instantaneous thermal energy (without losses) over this time period can then be estimated as $2 \times 10^{27}$ erg (calculation detailed in Hannah et al. 2008). Depending on whether thermal or non-thermal energies dominate the RHESSI lowenergy component during the first impulsive peak, this thermal energy is either a 4 or 20 times smaller than the non-thermal input. The difficulties in determining whether the low energy emission is thermal or non-thermal is partially due to RHESSI's degraded performance due to radiation damage. This should be rectified with the November 2007 anneal and so 2008 onwards flare studies should benefit from a reinvigorated RHESSI, as well as newer instruments such as Hinode. The microflares of AR10923 however are still very interesting, with further analysis and modeling required for these microflares, especially into how electrons were accelerated to such high energies in these small flares.

Acknowledgements. NASA supported this work under grant NAS5-98033 and NNG05GG16G. Hinode is a Japanese mission developed and launched by ISAS/JAXA, with NAOJ as domestic partner and NASA and STFC (UK) as international partners. It is operated by these agencies in co-operation with ESA and NSC (Norway).

\section{References}

Abbett, W. P., \& Hawley, S. L. 1999, ApJ, 521, 906

Bastian, T. S., Benz, A. O., \& Gary, D. E. 1998, ARA\&A, 36, 131

Bougeret, J.-L., Goetz, K., L., K. M., et al. 2007, Space Sci. Rev., in press Bougeret, J.-L., Kaiser, M. L., Kellogg, P. J., et al. 1995, Space Sci. Rev., 71, 231

Brosius, J. W., \& Phillips, K. J. H. 2004, ApJ, 613, 580

Brown, J. C. 1971, Sol. Phys., 18, 489

Dennis, B. R., \& Zarro, D. M. 1993, Sol. Phys., 146, 177

Farnik, F., Hudson, H., \& Watanabe, T. 1997, A\&A, 320, 620

Fisher, G. H., Canfield, R. C., \& McClymont, A. N. 1985, ApJ, 289, 434

Golub, L., DeLuca, E., Austin, G., et al. 2007, Sol. Phys., 243, 63

Handy, B. N., Acton, L. W., Kankelborg, C. C., et al. 1999, Sol. Phys., 187, 229

Hannah, I. G., Christe, S., Krucker, S., et al. 2008, ApJ, in press

Hudson, H. S., \& Ohki, K. 1972, Sol. Phys., 23, 155

Kosugi, T., Matsuzaki, K., Sakao, T., et al. 2007, Sol. Phys., 243, 3

Krucker, S., Hannah, I. G., \& Lin, R. P. 2007, ApJ, in press

Lin, R. P., Anderson, K. A., Ashford, S., et al. 1995, Space Sci. Rev., 71, 125

Lin, R. P., Dennis, B. R., Hurford, G. J., et al. 2002, Sol. Phys., 210, 3

McDonald, L., Harra-Murnion, L. K., \& Culhane, J. L. 1999, Sol. Phys., 185

Milligan, R. O., Gallagher, P. T., Mathioudakis, M., et al. 2006, ApJ, 642, L169

Neupert, W. M. 1968, ApJ, 153, L59

Phillips, K. J. H. 2004, ApJ, 605, 921

Sui, L., Holman, G. D., \& Dennis, B. R. 2006, ApJ, 645, L157

Sui, L., Holman, G. D., \& Dennis, B. R. 2007, ApJ, 670, 862

Veronig, A. M., Brown, J. C., Dennis, B. R., et al. 2005, ApJ, 621, 482 